\title{
Docentes vítimas de violência laboral e a implicação nas dimensões da síndrome de Burnout
}

\section{Teachers victims of workplace violence and the implications of the Burnout syndrome dimensions}

Beatriz Maria dos Santos Santiago Ribeiro ${ }^{1}$, Júlia Trevisan Martins ${ }^{2}$, Rita de Cassia de Marchi Barcelos Dalri ${ }^{3}$, Aline Aparecida Oliveira Moreira ${ }^{4}$

1. ORCID: https://orcid.org/0000-0001-5211-5422. Mestre em Enfermagem. Doutoranda em Ciências da Saúde na Escola de Enfermagem (Universidade de São Paulo, Ribeirão Preto, São Paulo, Brasil). E-mail: beatrizsantiago1994@hotmail.com.

2. ORCID: https://orcid.org/0000-0001-6383-7981. Doutora em Enfermagem. Docente do Curso de Graduação em Enfermagem e da Pós-Graduação Stricto Sensu (Universidade Estadual de Londrina, Londrina, Paraná, Brasil). E-mail: jtmartins@uel.br

3. ORCID: https://orcid.org/0000-0002-6575-5426. Doutora. Docente do Curso de da Pós-Graduação Stricto Sensu Escola de Enfermagem de Ribeirão Preto (Universidade de São Paulo, Ribeirão Preto, São Paulo, Brasil). E-mail: ritacmbdalri@bol.com.br

4. ORCID: https://orcid.org/0000-0002-2621-7078. Mestre em Enfermagem. Doutoranda em Enfermagem (Universidade Estadual de Londrina, Londrina, Paraná, Brasil). E-mail: alineaoliveira@yahoo.com.br

CONTATO: Nome do autor correspondente: Beatriz Maria dos Santos Santiago Ribeiro | Endereço: Sítio São Joaquim. Laranjal, Califórnia- PR. Telefone: 43 9.91724895 - E-mail: beatrizsantiago1994@hotmail.com 
RESUMO O presente estudo teve como objetivo comparar variáveis de violência fisica e verbal e sua implicação nas dimensões do burnout. Tratase de um estudo exploratório, cuja a população foi docentes do ensino fundamental e médio de escolas públicas de um município de médio porte localizado no Sul do Brasil. Foi utilizado o teste de ' $U$ ' de Mann-Whitney para comparação de médias. A maior parte dos participantes eram do sexo feminino, as idades variaram de 21 a 66 anos. Os docentes que sofreram violência física e verbal no ambiente laboral nos últimos 12 meses, apresentaram níveis maiores de exaustão emocional e despersonalização contudo a realização profissional houve pouca alteração, a presença de duas dimensões alteradas já indica síndrome de Burnout. A violência contra os docentes no ambiente escolar prejudica a saúde dos profissionais e consequentemente o desempenho profissional.

DESCRITORES: Docentes. Violência no trabalho. Esgotamento Psicológico.

ABSTRACT The present study aimed to compare variables of physical and verbal violence and its implication in the burnout dimensions. This is an exploratory study, whose population was teachers of elementary and high school in public schools in a medium-sized municipality located in southern Brazil. The Mann-Whitney ' $U$ ' test was used to compare means. Most participants were female, ages ranged from 21 to 66 years. Teachers who suffered physical and verbal violence in the workplace in the past 12 months, had higher levels of emotional exhaustion and depersonalization; however, professional fulfillment was little changed, the presence of two altered dimensions already indicates Burnout syndrome. Violence against teachers in the school environment harms the professionals health and consequently their professional performance

DESCRIPTORS: Faculty Teachers. Workplace Violence. Burnout, Psychological. 


\section{INTRODUÇÃO}

1 violência no ambiente laboral é um problema de saúde emergente e abrange diferentes áreas de atuação, também é marcada como um problema social e histórico que alcança as escolas ${ }^{1}$.

As violências sofridas no ambiente laboral levam os docentes à desmotivação e insatisfação, prejudicando o desenvolvimento de suas atividades, isto é, contribuem para uma educação de baixa qualidade ${ }^{2}$. Também provocam danos ao desenvolvimento subjetivo e social do aluno, bem como afeta a saúde física e psíquica do professor ${ }^{3}$. A violência física contribui para a insatisfação no trabalho ${ }^{4}$ e provoca a sensação de insegurança e medo de sofrer violência futura ${ }^{5}$.

Enfatiza-se que sofrer atos de violência pode provocar danos à saúde e afetar a integridade física e psíquica dos trabalhadores, provocando sinais e sintomas de natureza psicossomática, podendo dar origem ou agravar doenças, alterações no sono, depressão, ansiedade, entre outros ${ }^{6}$.

Entre as doenças ocupacionais de ordem mental, encontra-se a síndrome de Burnout que é caracterizada pelo esgotamento físico e mental decorrente de estresse vivenciado no labor, sendo descrita e analisada no início da década de 1970, pelo médico psiquiatra Freudenberg ${ }^{7}$. A síndrome de Burnout é uma resposta prolongada aos estressores crônicos emocionais e interpessoais envolvidos na atividade laboral, acarreta variados prejuízos familiares, pessoais, profissionais e sociais ${ }^{8}$.

Salienta-se que tal síndrome atinge profissionais de diferentes áreas, tais como a saúde, educação, serviço social, justiça e outras; de forma geral atinge profissões que se relacionam diretamente com o ser humano, que ao longo do tempo se desgastam devido a fatores como pouca remuneração, falta de reconhecimento, pela desmotivação, pelas relações interpessoais conflituosas, dentre outros fatores ${ }^{8}$.

A presente pesquisa se justifica mediante à escassez de estudos sobre violência escolar na realidade brasileira, principalmente de caráter quantitativo. Estudo de revisão sistemática realizado em 2014 identificou apenas quatro textos com análises quantitativas de dados sobre as violências imputadas contra o professor ${ }^{9}$.

Perante o cenário brasileiro, acredita-se que este estudo seja de fundamental importância; ressalta-se o docente como um ator imprescindível no processo da educação e a violência ocupacional pode interferir nesse processo e gerar problemas graves a nível nacional, incluindo diminuição no número de docentes. Ressalta-se a importância dos docentes para a formação da sociedade e se faz necessário que eles estejam motivados e engajados. A coleta de dados foi realizada em um município de médio porte e tais municípios geralmente são poucos pesquisados. Acredita-se que 
em localidades menores a infraestrutura e condições de trabalho sejam diferentes de cidades de grande porte.

Diante do exposto, elaborou-se a seguinte questão de pesquisa: Os docentes do ensino fundamental e médio de escolas públicas que vivenciaram a violência fisica e verbal no trabalho, apresentam indicativos para a síndrome de Burnout? Para responder esse questionamento essa pesquisa teve como objetivo comparar variáveis de violência fisica e verbal e sua implicação nas dimensões do burnout.

\section{MÉTODO}

Estudo exploratório, realizado com docentes do ensino fundamental e médio de escolas públicas de um município de médio porte localizado no Sul do Brasil. Para a coleta de dados, foram convidados docentes que sofreram violência física e/ou verbal nos 12 meses anteriores a coleta de dados.

Para a coleta de dados, utilizou-se um instrumento para obtenção de dados laborais e o questionário de Avaliação da Violência no Trabalho Sofrida ou Testemunhada por Trabalhadores de Enfermagem nos últimos 12 meses $^{10}$, sendo adaptado no Brasil para uso com docentes. Após a autorização das autoras, acrescentou-se no cabeçalho do referido instrumento os conceitos de violência e, posteriormente, ele foi enviado para sete juízes especialistas na temática ou na validação de instrumentos de diferentes áreas sendo três educadores, dois psicólogos, um assistente social e um advogado.

A síndrome de Burnout foi avaliada pelo Maslach Inventory Burnout- Human Services Survey (MBI-HSS), instrumento criado no ano de 1973, fundamentado em um programa de pesquisa psicométrica denominado Maslach Burnout Inventory (MBI) de autoria de Cristina Maslach, está validado em diversos países, bem como em vários idiomas e é tido como um dos mais utilizados em todo mundo para avaliar o burnout. O MBI-HSS avalia três dimensões da experiência de Burnout por meio de 22 questões em escala Likert com respostas que pontuam de zero a seis pontos, dentre as quais nove avaliam a exaustão emocional (EE), cinco avaliam a despersonalização (D) e oito avaliam a realização profissional $(\mathrm{RP})^{8}$. As licenças para a utilização do MBI-HSS foram adquiridas na empresa Mind Garden, a qual administra os direitos autorais do instrumento.

A coleta ocorreu entre os meses de julho a novembro de 2018, por meio eletrônico com a utilização da plataforma Google Forms. Determinou-se a prevalência da síndrome de Burnout considerando os seguintes pontos de corte: nível alto $\geq 26$ para exaustão emocional, nível alto $\geq 9$, despersonalização e baixa realização profissional $\leq 33$. Assim, se considera uma pessoa com burnout se houverem altos níveis para exaustão emocional e despersonalização e nível baixo para realização profissional. Autores mencionam que 
somente as duas primeiras dimensões já são suficientes, quando alteradas para indicar burnout $^{11}$, portanto no presente estudo, optou-se em considerar, principalmente as duas primeiras dimensões.

A consistência interna do MBI-HSS foi avaliada por meio do cálculo do coeficiente alfa de Cronbach. Foi aplicado teste ' $U$ ' de Mann-Whitney com a finalidade de comparar as médias entre as subcategorias, visto que as variáveis não apresentaram distribuição normal. As hipóteses testadas foram: $\mathrm{H}_{0}$ : as médias da população são todas iguais e $\mathrm{H}_{1}$ : as médias da população não são todas iguais. A pesquisa foi apreciada por Comitê de Ética em Pesquisa, obtendo aprovação com Certificado de Apresentação para Apreciação Ética (CAAE) 87890218.4.0000.5231 e número do Parecer 2.744.982.

\section{RESULTADOS}

Dos participantes, 81 docentes $(71,7 \%)$ eram do sexo feminino e $32(28.3 \%)$ do sexo masculino, apresentando idade entre 21 e 66 anos. Quanto à consistência interna do MBIHSS, este apresentou valor satisfatório com $\alpha=0,83$, caracterizando boa consistência interna e confiabilidade.

Sofrer violência nos turnos da manhã e tarde, apresentaram nível mais elevado de síndrome de Burnout. Os docentes que não registraram o episódio da violência tiveram níveis mais elevados respectivamente com $\mathrm{EE}(\mu=37,9), \mathrm{D}(\mu=14,56)$, RP $(\mu=32,42)$. No que tange as consequências para o agressor, as variáveis obtiveram diferenças significativas quando o agressor não teve punição ou recebeu a advertência verbal (Tabela 1). 
Tabela 1: Comparação das dimensões do burnout entre os docentes investigados e a violência verbal ocupacional entre os docentes investigados.

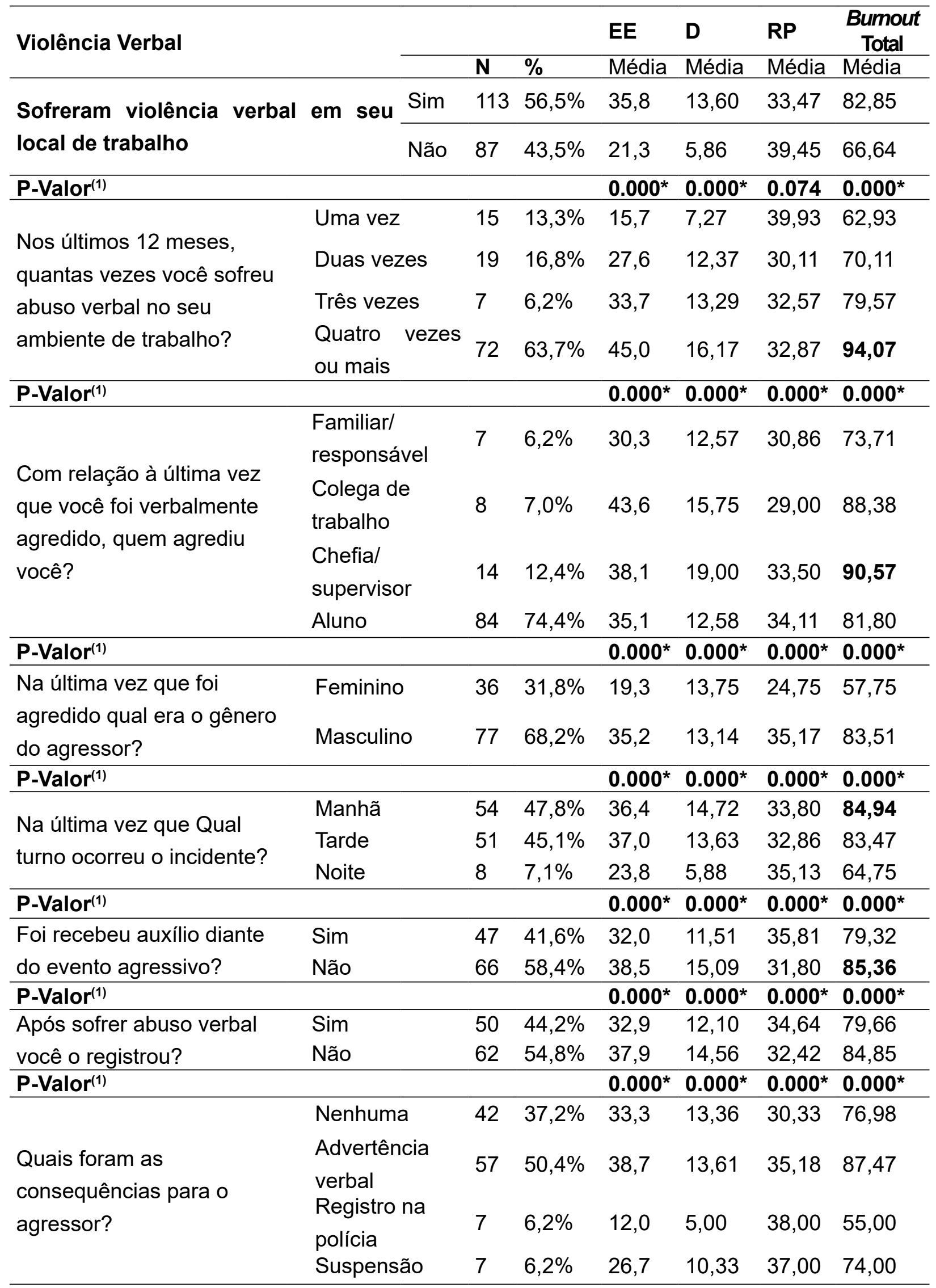

Fonte: Elaborado pelos autores (2018). 
(1) Teste 'U' de Mann-Whitney (p-valor<0.05).

*Valores Significativos; NS - Valores Não Significativos.

Interpretação do teste:

$\mathbf{H}_{\mathbf{0}}$ : As médias observadas ocorrem na mesma proporção nos grupos.

Ha: As médias observadas diferem significativamente entre os grupos.

Decisão: Como o valor de $p$ computado é menor que o nível de significância alfa $=0,05$, deve-se rejeitar a hipótese nula $\mathrm{H}_{0}$ e aceitar a hipótese alternativa $\mathrm{Ha}$.

Nota: EE - Exaustão Emocional; D - Despersonalização; RP - Realização Pessoal

Denota-se que se observou no estudo inferência significativa das variáveis maiores de exaustão emocional e despersonalização, porém, pouca inferência significativa com a realização profissional.

Em relação a violência física somente seis docentes (3\%) responderam afirmativamente. Os alunos foram os agressores em $100 \%$ dos casos. Em relação entre as médias obtidas pelos docentes em função da quantidade de episódios de violência física vivenciada, de forma que os docentes que sofreram um ou dois episódios de violência física nos últimos 12 meses, apresentaram diferença significativa no escore médio das dimensões de EE e $D$, indicando níveis de burnout.

Observa-se que docentes agredidos pelos alunos do sexo feminino apresentaram níveis mais elevados de burnout com EE $(\mu=54)$ e $D(\mu=24)$, RP $(\mu=15)$. O mesmo ocorreu em docentes que atuavam no turno da manhã que apresentaram alteração nas dimensões com EE $(\mu=44)$, D $(\mu=18)$ e RP $(\mu=28)$ (Tabela 2).

Os docentes cujo agressor recebeu advertência verbal tiveram alterações nas dimensões EE $(\mu=51), D(\mu=20)$ e RP $(\mu=28)$ e de forma geral, apresentaram escore médio mais elevado ( $\mu=99)$ (Tabela 2 ).

Ressalta-se que não foi possível verificar outros aspectos na categoria de violência física, devido à baixa prevalência do desfecho, podendo acarretar dados frágeis.

Os docentes que sofreram abuso verbal no seu ambiente de trabalho por duas vezes $(\mu=108)$, apresentaram níveis elevados para a síndrome de Burnout (Tabela 2).

No aspecto geral, independente de quem seja o agressor há aumentos em dimensões como encontrado na categoria de quem agrediu você. Em relação ao sexo feminino houve maiores alterações totalizando $(\mu=93)$ (Tabela 2$)$. 
Tabela 2: Comparação das dimensões do burnout entre os docentes investigados e a violência física ocupacional entre os docentes investigados.

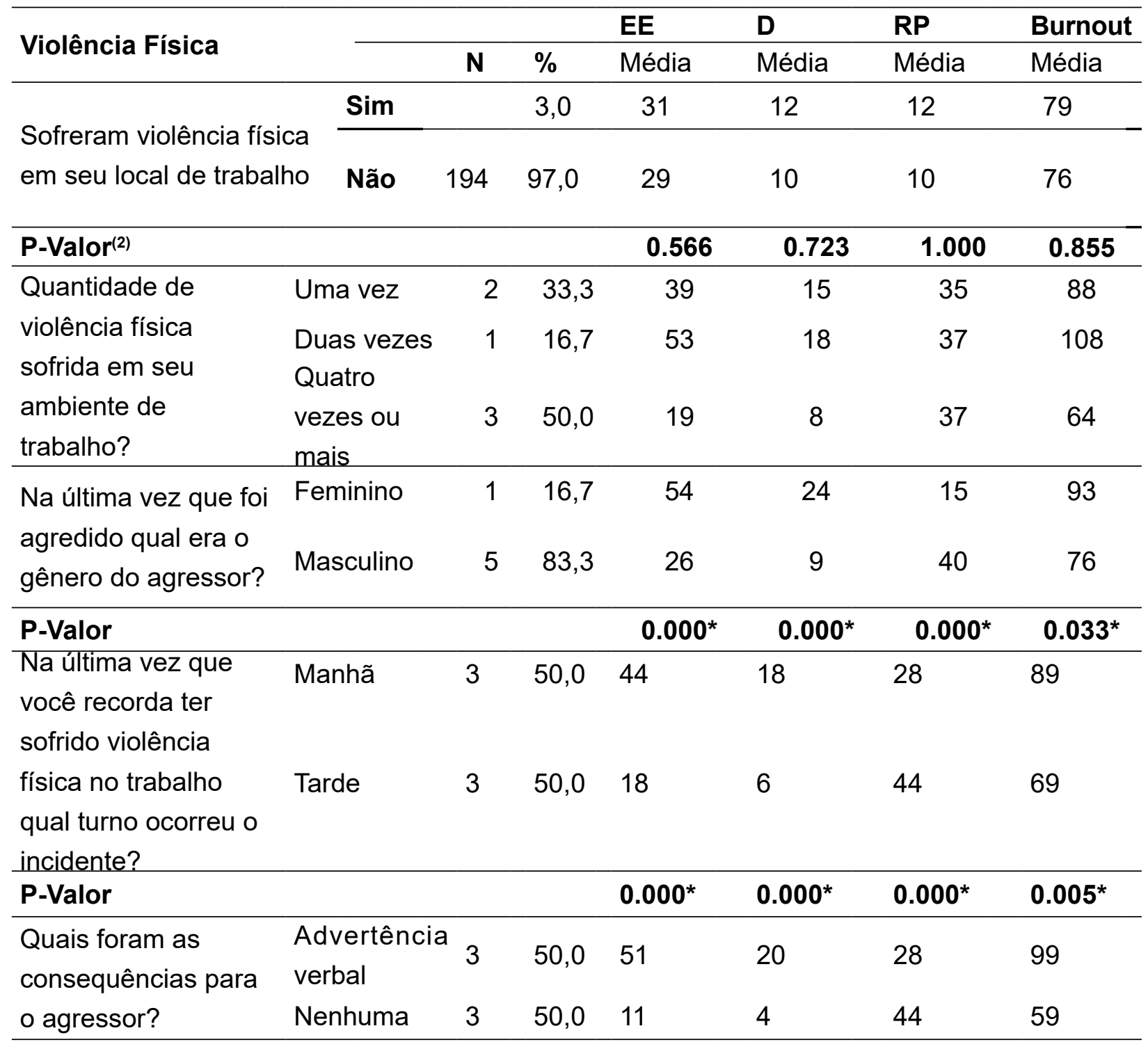

Fonte: Elaborado pelos autores (2018).

(1) Teste 'U' de Mann-Whitney ( $p$-valor<0.05).

*Valores Significativos; NS - Valores Não Significativos.

Interpretação do teste:

$\mathbf{H}_{0}$ : As médias observadas ocorrem na mesma proporção nos grupos.

Ha: As médias observadas diferem significativamente entre os grupos.

Decisão: Como o valor de $p$ computado é menor que o nível de significância alfa $=0,05$, deve-se rejeitar a hipótese nula $\mathrm{H}_{0}$ e aceitar a hipótese alternativa $\mathrm{Ha}$.

Nota: EE - Exaustão Emocional; D - Despersonalização; RP - Realização Pessoal 


\section{DISCUSSÃO}

A violência ocupacional reflete na saúde física e mental dos trabalhadores ${ }^{12}$, bem como no bem-estar psicológico ${ }^{13}$, como foi constatado nesse estudo em que professores apresentaram alterações nas dimensões da síndrome de Burnout. Essa síndrome afeta negativamente seu desempenho, pois prejudica o alcance dos objetivos pedagógicos e o ambiente escolar levando os educadores a experimentarem um processo de apatia, alienação, sofrimento psíquicos e problemas de saúde ${ }^{14}$. O presente estudo identificou que as violências ocupacionais interferem nos níveis da exaustão emocional e despersonalização, mas não interferiu significativamente nas variáveis relacionadas à terceira dimensão da síndrome burnout, ou seja, a realização profissional.

A violência é uma resposta negativa e desmotivada ao trabalho e prejudica a saúde mental dos trabalhadores ${ }^{15}$; outros autores comprovaram que a síndrome de Burnout, surge da violência ocupacional que os funcionários vivenciam ${ }^{16}$. Ainda, a violência no trabalho é um preocupante estressor social que esgota tanto os recursos emocionais como os físicos, levando ao burnout.16. Desse modo, os resultados apresentados nessa pesquisa corroboram com os estudos apresentados acima, evidenciando que os níveis do burnout estão associados com a exposição à violência.

Acredita-se que docentes que sofrem mais de quatro ou mais tentativas de violência criam mecanismos de resiliência, visto que não houve diferenças nas médias obtidas.

Os resultados da amostra pesquisada com os docentes, mostraram que ocorreu associação entre a violência e a síndrome de Burnout, dados que coincidem com estudo realizado na Polônia com 1.214 docentes, que demonstrou que vivenciar comportamentos agressivos na escola levam a sintomas de burnout ${ }^{17}$. Em terras brasileiras, examinou-se a exposição entre tolerar insultos de alunos com a dimensão de exaustão emocional, constatando a relação da violência com a síndrome de Burnout ${ }^{18}$.

Nesse estudo os docentes foram agredidos verbalmente por familiares e responsáveis. Denota-se em concordância com um estudo, que uma parcela significativa dos alunos e seus pais, enxergam a escola como um agente de exclusão, que transforma a experiência dos alunos e abre uma crise, o que propicia espaço a desvalorização do professor, e, muitas vezes, da legitimidade da instituição ${ }^{19}$. Ressalta-se que a violência pode provocar prejuízos a todos envolvidos, afeta à saúde física e psíquica do professor e o desenvolvimento subjetivo e social do aluno ${ }^{3}$.

O que chama atenção é que o docente pode ficar exposto às violências físicas e verbais, produzindo desiquilíbrio entre esforço-recompensa que na maioria das vezes é desproporcional em relação ao plano de carreira, reconhecimento público e remuneração, mostrando propensão a desenvolver problemas de natureza mental ${ }^{20}$. O burnout ocasiona inúmeras consequências ao trabalhador desde dores musculoesqueléticas, insônia, 
fadiga prolongada e sintomas depressivos ${ }^{21}$ e exaustão emocional e despersonalização conforme os resultados observados deste estudo.

Investigação realizada nos Estados Unidos com 2.000 docentes que desenvolviam atividades em diferentes níveis de ensino, mostrou que $80 \%$ desses profissionais afirmaram ter tido pelo menos uma experiência de violência, sendo que $94 \%$ foram impetradas por alunos e $44 \%$ afirmaram terem sido agredidos fisicamente ${ }^{22}$.

Ressalta-se que há necessidade de estabelecer um relacionamento interpessoal entre docente-discente, pois um estudo mostrou que os alunos consideraram um bom professor, aquele profissional que além de ministrar os conteúdos, estimula a participação do aluno buscando uma relação dialógica com os mesmos. É fato que a existência de coerência nas atitudes do docente e quanto mais autêntico ele for nas relações, maior será a possibilidade de envolver-se positivamente com o aluno e por consequência, um melhor ensino-aprendizado ocorrerá23. Pode-se inferir que uma boa relação interpessoal pode ser uma ferramenta importante para diminuir atos de violência na escola, principalmente nos casos de violência física em que o aluno foi agressor em todos casos deste estudo.

Se tratando da saúde do trabalhador, uma pesquisa trouxe que $42 \%$ dos docentes entrevistados mencionaram dor em alguma parte do corpo há mais de seis meses, relataram sobrecarga do trabalho com elevadas jornadas laborais desenvolvidas na posição em pé, posturas ante ergonômicas, aspectos psicossociais não favoráveis, sendo estes relatos facilitadores para a dor crônica $^{24}$. Alterações frequentes do sono que resultam em fadiga, prejuízo no desempenho laboral e consequentemente piora na qualidade de vida foram apresentadas em outra investigação ${ }^{25}$.

Pesquisa realizada em Viçosa (MG) ${ }^{26}$, apontou que $40 \%$ dos docentes da rede pública entrevistados se ausentaram do trabalho por motivo de saúde e em destaque, o motivo mais comum foi o estresse devido às más condições laborais e baixos salários.

Percebe-se que as doenças psíquicas tiveram grande aumento em trabalhadores que desenvolvem suas atividades em empresas, todavia, é preocupante no cenário do professor, tendo em vista a verificação do alto índice de absenteísmo por doenças mentais como a síndrome de Burnout. Verificou-se que na classe dos trabalhadores educacionais há um impacto das condições de trabalho, o que se torna um fator de risco para o burnout ${ }^{27}$.

Ocorre com maior predominância da agressão ser perpetrada pelo sexo masculino ${ }^{28}$, assim como abordado nesse estudo que o gênero masculino foi o mais recorrente como agressor tanto em violência física quanto verbal.

Em 1999, foi realizado um estudo com professores do ensino fundamental e médio em todo o país, com 30 mil docentes e revelou-se que $26 \%$ apresentou exaustão emocional. Decorreram mais de 20 anos e ainda prevalece o mesmo problema com esse público ${ }^{29}$. 
Com isso, os autores ${ }^{30}$, mostraram em seu estudo a importância do psicólogo atuar nas escolas públicas, devido ao estresse e a violência laboral impetrada contra os docentes e ainda revelaram que sentimento de insegurança e os problemas emocionais que os docentes enfrentam em situações de violência, podem provocar desgaste, desmotivação e, por sua vez, levá-los ao adoecimento mental, já que nessa pesquisa, os profissionais que vivenciaram a violência física e verbal, sofreram interferência nos níveis de Burnout.

Ressalta-se que relações interpessoais quando há desgaste, podem gerar elevado percentual de violência no trabalho, o que precisa ser monitorado e medidas devem ser discutidas para o enfrentamento dessa situação. Como limitação do estudo, aponta-se o fato de ter sido desenvolvido em apenas um município de médio porte, contudo permite comparações com população semelhante em estudos futuros.

Acreditamos que o exposto nesse trabalho pode contribuir para o avanço em novos conhecimentos, visto que o exposto apresenta uma possibilidade de novos estudos sobre a temática de violência e agravos a saúde dos docentes para a realidade brasileira. Sendo assim, quanto maior o cuidado a saúde do docente, maior será o retorno para sociedade.

\section{CONCLUSÃO}

Nesse estudo os docentes que estiveram expostos à violência física e verbal no trabalho nos últimos 12 meses anteriores à coleta de dados, apresentaram níveis maiores de exaustão emocional e despersonalização, porém na realização profissional, houve pouca alteração, assim as duas primeiras dimensões alteradas são indicativos da Síndrome de Burnout. Destaca-se que conhecer a exposição da violência no trabalho docente, contribui não somente para o conhecimento da violência no ambiente escolar, mas permite a busca de subsídios de âmbito social.

Aponta-se que há necessidade da criação de políticas protetoras que facilitem que docentes, alunos, sociedade e gestores busquem em conjunto ações para extinguir/ minimizar a violência e os agravos de saúde aos docentes vitimizados, visando consequentemente melhora da qualidade de vida laboral e menos adoecimentos físicos e mentais.

\section{REFERÊNCIAS}

1. Chappell D, Di Martino V. Violence at work. Third edition. [Internet] Geneva: ILO; 2006.

2. Gurgel CR, Matos FAS. A violência contra professores: saberes e práticas. Campina Grande: Realize Editora, 2012.

3. Brasil KCT, Legnani VN, Vieira RA. Violência 'na' e 'da' escola: concepções de professores e alunos adolescentes. Linhas Críticas. 2015 set./dez. [acesso em 2019 jul 10]; (46):708726. Disponível em: https:// www.redalyc.org/pdf/1935/193543849008.pdf 
4. Giorgi, G, Mancuso S, Perez FF, D'Antonio AC, Mucci N, Cupelli V et al. Bullying among nurses and its relationship with burnout and organizational climate. International journal of nursing practice [Internet]. 2016 [acesso em 2019 jul 01]; (22):160-168. Disponível em: https://doi.org/10.1111/ijn.12376

5. Portoghese I, Galletta M, Leiter MP, Cocco P, D'Aloja E, Campagna M. Fear of future violence at work and job burnout: A diary study on the role of psychological violence and job control. Burnout Research. 2017 [acesso em 2019 jul 15]; (7):36-46. Disponível em: https://doi.org/10.1016/j.burn.2017.11.003.

6. Sousa AJA. Campanha de comunicação - violência no trabalho (Trabalho de Projeto). Instituto Politécnico de Lisboa, Escola Superior de Comunicação Social, Lisboa, 2013.

7. Guimarães TCP, Manhães FCA. Síndrome de Burnout: Um Estudo Comparativo Sobre APrática Pedagógica E A Qualidade Do Ensino Entre Docentes Que Atuam Na Educação De Jovens, Adultos E Idosos E Na Modalidade Regular. Revista Científica Interdisciplinar. 2015 [acesso em 2019 jun 30]; 2(3):112-6. Disponível em: http://revista.srvroot.com/linkscienceplace/index.php/linkscienceplace/article/view/145

8. Maslach C, Schaufeli WB, Leiter MP. Job burnout. Ann. rev. clin. psychol. 2001 [acesso em 2019 mar 15]; 52:397-422. Disponível: https://doi.org/10.1146/annurev.psych.52.1.397

9. Nesello F, Sant'Anna FL, Santos HGD, Andrade SMD, Mesas AE, González AD. Características da violência escolar no Brasil: revisão sistemática de estudos quantitativos. Rev. Bras. Saude Mater. Infant. 2014 [acesso em 2019 fev 20]; 14(2):119-136. Disponível: https://doi.org/10.1590/S1519-38292014000200002.

10. Bordignon M, Monteiro Ml. Validade aparente de um questionário para avaliação da violência no trabalho. Acta paul. enferm. 2015 [acesso em $2019 \mathrm{fev}$ 20]; 28(6). Disponível em: https://doi. org/10.1590/1982-0194201500098

11. Demerouti E, Bakker AB, Vardakou I, Kantas A. The convergent validity of two burnout instruments: a multitrait-multimethod analysis. Eur. j. psychol. assess. 2003; 19(1):12.

12. Miranda H, Punnett L, Gore RJ. Musculoskeletal pain and reported workplace assault: a prospective study of clinical staff in nursing homes. Hum. factors. 2014 [acesso em 2019 abr 02]; 215-227. Disponível: https://doi.org/10.1177/0018720813508778

13. Verkuil B, Atasayi S, Molendijk ML. Workplace bullying and mental health: a meta-analysis on crosssectional and longitudinal data. PloS one. 2015 [acesso em 2019 abr 02]; 10:e0135225. Disponível em: https://doi.org/10.1371/journal.pone.0135225

14. DiehI L, Carlotto MS. Síndrome de Burnout: indicadores para a construção de um diagnóstico. Psicologia Clínica. 2015; 27(2):161-179.

15. Hamdan M, Hamra AA. Burnout among workers in emergency Departments in Palestinian associated factors. BMC health serv. res. (Online). 2017 [acesso em 2019 abr 24]; 17(1). Disponível em: https://doi. org/10.1186/s12913-017-2356-3

16. Livne Y, Goussinsky R. Workplace bullying and burnout among healthcare employees: The moderating effect of control-related resources. Nursing \& health sciences Epub ahead of print. 2017 [acesso em 2019 jul 13]. Disponível em: https://doi.org/10.1111/nhs.12392

17. Mościcka-Teske A, Drabek M, Pyżalski J. Experienced bullying and hostile behavior in the workplace and symptoms of burnout in teachers. Med. pracy. 2014 [acesso em 2019 jul 13]; (65):535-542. Disponível: https://doi.org/10.13075/mp.5893.00017

18. Koga GKC, Melanda FN, Santos HGD, Sant'Anna FL, González AD, Mesas AE et al. Fatores associados a piores níveis na escala de Burnout em professores da educação básica. Cad. saúde coletiva. 2015 [acesso em 2019 jul 22]; 23(3): 268-275. Disponível: https://doi.org/10.1590/1414-462X201500030121

19. Dubet F. A escola e a exclusão. Cad. pesqui. 2003; (119):29-45.

20. Van Droogenbroeck F, Spruyt B. Do teachers have worse mental health? Review of the existing comparative research and results from the Belgian Health Interview Survey. Teaching and Teacher Education. 2015 Oct 1 [acesso em 2019 jul 01]; 51:88-100. Disponível em: https://doi.org/10.1016/j.tate.2015.06.006

21. Salvagioni, DAJ, Melanda FN, Mesas AE, González AD, Gabani FL, Andrade SMD. Physical, psychological and occupational consequences of job burnout: A systematic review of prospective studies. PloS one. 2017 [acesso em 2019 jul 02]; 12(10): e0185781. Disponível em: https://doi.org/10.1371/journal. pone. 0185781

22. Espelage D, Anderman EM, Brown VE, Jones A, Lane KL, McMahon SD et al. Understanding and preventing violence directed against teachers: recommendations for a national research, practice, and policy agenda. Am Psychol. 2013 [acesso em 2019 jul 10]; (68):75-87. Disponível em: https://doi.org/10.1037/ a0031307 
23. Soares MH, Bueno SMV. Cenário do ensino do enfermeiro psiquiátrico. Ciênc. cuid. saúde. 2012 [acesso em 2019 jun 30]; 11(5):160-167. Disponível: https://doi.org/10.4025/cienccuidsaude.v11i5.17071

24. Silva AD. Prevalência e fatores associados à dor crônica em professores da rede estadual de LondrinaPR. 2014. 101 f. Dissertação (Mestrado em Saúde Coletiva) - Universidade Estadual de Londrina, Londrina, 2014.

25. Leme LRL. Qualidade de sono em indivíduos com dor crônica. 2014. 70 f. Dissertação (Mestrado em Enfermagem) - Centro de Pós-Graduação e Pesquisa, Universidade Guarulhos, Guarulhos, 2014.

26. Esteves-Ferreira AA, Santos DE, Rigolon RG. Avaliação comparativa dos sintomas da síndrome de burnout em professores de escolas públicas e privadas. Rev. bras. educ. 2014, 19(59):987-1002.

27. Carlotto MS, Câmara SG. Análise fatorial do Maslach Burnout Inventory (mbi) em uma amostra de professores de instituições particulares. Psicol. estud. 2004 [acesso em 2019 ago 05]; 9(3):499-505. Disponível: https://doi.org/10.1590/S1413-73722004000300018

28. Tavares PA, Pietrobom FC. Fatores associados à violência escolar: evidências para o Estado de São Paulo Estud. Econ. 2016 [acesso em 2019 ago 15]; 46(2):471-498. Disponível em: https://doi. org/10.1590/0101-416146277ptf

29. Codo W. Educação: carinho e trabalho. Petrópolis: Vozes, 1999.

30. Costa MSGA, Barbosa NDE, Carraro PR. A importância do trabalho do psicólogo escolar aos docentes em escolas públicas. Rev. EIXO. 2014 [acesso em 2019 ago 16]; 3(2): 73-80. Disponível em: https://doi. org/10.19123/eixo.v3i2.146 